\title{
ON THETA PAIRS FOR A MAXIMAL SUBGROUP
}

\author{
N. P. MUKHERJEE AND PRABIR BHATTACHARYA
}

(Communicated by Warren J. Wong)

\begin{abstract}
For a maximal subgroup $M$ of a finite group $G$, a $\Theta$-pair is any pair of subgroups $(C, D)$ of $G$ such that (i) $D \triangleleft G, D \subset C$, (ii) $\langle M, C\rangle=G$, $\langle M, D\rangle=M$ and (iii) $C / D$ has no proper normal subgroup of $G / D$. A natural partial ordering is defined on the family of $\Theta$-pairs. We obtain several results on the maximal $\Theta$-pairs which imply $G$ to be solvable, supersolvable, and nilpotent.
\end{abstract}

\section{INTRODUCTION}

There has been some interest in the past in investigating how some conditions imposed on a maximal subgroup of a finite group influence the structure of the group. Our objective is to associate a certain family of pairs of subgroups with any maximal subgroup and study how some conditions on the maximal elements (with respect to a natural partial ordering) of such a family imply that the group is solvable, supersolvable, or nilpotent. The family of subgroups we introduce is motivated by the interesting concept of the Index Complex defined in Deskins [4-5].

Definition. Given a maximal subgroup $M$ of a group $G$, let

$$
S_{M}=\{(A, B): A \leq G, B \triangleleft G, B \subset A,\langle M, A\rangle=G,\langle M, B\rangle=M\} .
$$

Also, let

$\Theta(M)=\left\{(C, D) \in S_{M}: C / D\right.$ contains properly no normal subgroup of $\left.G / D\right\}$. We call any pair $(C, D)$ in $\Theta(M)$ a $\Theta$-pair.

A partial order $\leq$ may be defined on $\Theta(M)$ as follows:

$$
(C, D) \leq\left(C^{\prime}, D^{\prime}\right) \text { if } C \leq C^{\prime} \text {; }
$$

no condition is placed on the second component of the pairs. (One notices that using the definition of $\Theta(M)$, it follows that $D \subset D^{\prime}$. Also, $C=C^{\prime} \Rightarrow D=$ $\left.D^{\prime}\right)$. It is easy to verify that

$$
(C, D) \leq\left(C^{\prime}, D^{\prime}\right) \text { and }\left(C^{\prime}, D^{\prime}\right) \leq(C, D) \Leftrightarrow C=C^{\prime}, D=D^{\prime} .
$$

Received by the editors June 2, 1989 and, in revised form, September 27, 1989.

1980 Mathematics Subject Classification (1985 Revision). Primary 20D10, 20D25; Secondary 20D99.

Key words and phrases. Solvable, supersolvable, nilpotent. 
Obviously, $\Theta(M)$ will contain maximal elements with respect to this ordering. We shall call a maximal element a maximal $\Theta$-pair. As a straightforward example, take $G=\operatorname{Sym}(4)$. Then if $M \in \operatorname{Syl}_{2}(G), \operatorname{Core}_{G} M$ is the Klein 4-group and it is easy to see that $(\operatorname{Sym}(3),\langle e\rangle) \in \Theta(M)$ and $(\operatorname{Sym}(3),\langle e\rangle)$ is a maximal $\Theta$-pair. For any group $G$ and a maximal subgroup $M$ of $G$, if $(C, D) \in \Theta(M)$, then clearly $D \subset \operatorname{Core}_{G}(M)$; but $D$ need not be equal to Core $_{G} M$ as the above example shows.

In $\S 3$ we shall obtain conditions on maximal pairs in $\Theta(M)$ which imply $G$ to be solvable, or supersolvable. In $\S 4$ we shall obtain conditions on maximal pairs in $\Theta(M)$ which imply $G$ to be nilpotent, or $p$-nilpotent.

All groups considered are finite. We use standard notation as in Huppert [7]. By a slight abuse of language, by a simple group we shall always mean a simple, non-Abelian group. Also, for convenience we denote $M \lessdot G$ to indicate that $M$ is a maximal subgroup of $G$. If $M \lessdot G$ and $[G: M]$ is composite, then $M$ is called a c-maximal subgroup of $G$.

\section{Preliminaries}

If $M<G$ and $\langle M, A\rangle=G$, then any normal subgroup $B$ of $G$ contained in $A$ with $\langle M, B\rangle=M$ will produce a pair $(A, B) \in S_{M}$. In particular, $(A,\langle e\rangle)$ where $e$ denotes the identity of $G$, is also a pair in $S_{M}$. If $(A, B)$ is any pair in $S_{M}$, then clearly $B \subset$ Core $_{G} M$.

If $M \lessdot G$, then $\Theta(M)$ is nonempty. For, let $C \leq G$ such that $\langle M, C\rangle=G$ and denote

$$
D=\prod\left\{Y:(C, Y) \in S_{M}\right\} .
$$

Then, if $C / D$ has no proper normal subgroup of $G / D$, one has that $(C, D) \in$ $\Theta(M)$. Otherwise, suppose that $L / D$ is a minimal normal subgroup of $G / D$ contained in $C / D$. Then it is easy to see that $(L, D) \in \Theta(M)$.

The following result will be used frequently in induction arguments.

Lemma 2.1. If $(C, D)$ is a maximal $\Theta$-pair in $\Theta(M)$ and $N \triangleleft G, N \subset D$, then $(C / N, D / N)$ is a maximal $\Theta$-pair in $\Theta(M / N)$. Conversely, if $(C / N, D / N)$ is a maximal $\Theta$-pair in $\Theta(M / N)$, then $(C, D)$ is a maximal $\Theta$-pair in $\Theta(M)$.

Proof. $\Rightarrow$ : Since $\langle M, C\rangle=G,\langle M, D\rangle=M$, and $C / D$ has no proper normal subgroup of $G / D$, it follows that $(C / N, D / N) \in \Theta(M / N)$. If $(C / N, D / N)$ is not a maximal $\Theta$-pair in $\Theta(M / N)$, then suppose that $(C / N, D / N) \leq$ $(X / N, Y / N), C / N \subset X / N$. This implies that $C \subset X$. Now, one sees that $(X, Y) \in \Theta(M)$. Further, $(C, D) \leq(X, Y)$, since $C \subset X$. This violates the maximality of $(C, D)$ in $\Theta(M)$.

$\Leftarrow$ : It is easy to see that $(C, D) \in \Theta(M)$. If $(C, D)$ is not a maximal $\Theta$-pair, then suppose $(C, D) \leq\left(C_{1}, D_{1}\right)$ and $C \subset C_{1}$. This implies that $(C / N, D / N) \leq\left(C_{1}, D_{1} / N\right)$, violating the maximality of the pair $(C / N, D / N)$. We have used here the fact that $N \subset D_{1}$. To see this, first we observe that $\left\langle C_{1}, M\right\rangle=G,\left\langle M, D_{1}\right\rangle=M$, and $N \subset C \subset C_{1}$. If $N \not \subset D_{1}$, then $N D_{1} / D_{1}$ 
is a normal subgroup of $G / D_{1}$, and this, since $\left(C_{1}, D_{1}\right) \in \Theta(M)$, means $N D_{1}=C_{1}$. However, in that case $G=\left\langle M, C_{1}\right\rangle=\left\langle M, N D_{1}\right\rangle=M$ since $\left\langle M, D_{1}\right\rangle=M$ and $N \subset D$, a contradiction.

\section{Solvability CONDITIONS}

Theorem 3.1. A group $G$ is solvable $\Leftrightarrow$ for each $M \lessdot G$, every maximal $\Theta$-pair $(C, D)$ in $\Theta(M)$ is such that $C / D$ is solvable.

Proof. $\Leftarrow$ : Obviously, the hypothesis cannot hold if $G$ is simple. Let $N$ be a minimal normal subgroup of $G$. We use induction on the order of $G$. If $M / N \lessdot G / N$ and $(C / N, D / N)$ is a maximal $\Theta$-pair in $\Theta(M / N)$, then by Lemma 2.1, $(C, D)$ is a maximal $\Theta$-pair in $\Theta(M)$. It follows by induction that $G / N$ is solvable, and without loss in generality, $N$ may be assumed to be the unique minimal normal subgroup of $G$. If $N \subseteq \Phi(G)$, the Frattini subgroup of $G$, then $G$ is solvable and therefore assume that $G=M_{1} N$ for some $M_{1} \lessdot G$. If $(N,\langle 1\rangle)$ is a pair in $\Theta(M)$ and if it is not a maximal $\Theta$-pair, then since $M_{1}$ is core-free, $\left.N,\langle 1\rangle\right) \leq(R,\langle 1\rangle)$ for some pair $(R,\langle 1\rangle)$ in $\Theta(M)$. But then $R /\langle 1\rangle$ has no proper normal subgroup of $G /\langle 1\rangle$, which is not possible since $N \subset R$. Thus $(N,\langle 1\rangle)$ is a maximal $\Theta$-pair in $\Theta\left(M_{1}\right)$, and so $N$ is solvable, implying that $G$ is solvable.

The converse holds trivially.

We remark that it can be shown that Theorem 3.1 remains valid if the statement " $C / D$ is solvable" is replaced by " $C / D$ is solvable whenever $C / D \triangleleft$ $G / D$ ". The proof of the following result is analogous to the proof of Theorem 3.1 and is omitted.

Theorem 3.2. (i) A group $G$ is solvable $\Leftrightarrow$ for each maximal pair $(C, D)$ in $\Theta(M), M \lessdot G$, one has that $C_{G / D}(C / D) \neq\langle\overline{1}\rangle$ whenever $C / D \unlhd G / D$.

(ii) A group $G$ is solvable if for every $M \lessdot G$, each maximal pair $(C, D)$ is such that $L(G / D) \neq\langle\overline{1}\rangle$.

(Here, for any group $X, L(X)$ denotes the intersection of all c-maximal subgroups of $X$; if there is no subgroup then set $L(X)=X([2-3]))$.

(iii) A group $G$ is solvable $\Leftrightarrow$ for any two distinct maximal subgroups $X$ and $Y$ of $G$ whenever $\Theta(X)$ and $\Theta(Y)$ have a common maximal pair $(C, D)$ it follows that $[G: X]=[G: Y]$ if $C / D \triangleleft G / D$.

We now give another characterization of solvable groups in terms of $\Theta$-pairs.

Theorem 3.3. A group $G$ is solvable $\Leftrightarrow$ for each c-maximal subgroup $M$ of $G$, there exists a maximal pair $(C, D)$ in $\Theta(M)$ such that $C / D$ is Abelian.

Proof. $\Leftarrow$ : Obviously, $G$ cannot be simple. Let $N$ be a minimal normal subgroup of $G$. We use induction on the order of $G$. If $R / N$ is a $c$-maximal subgroup of $G / N$, then $R$ is $c$-maximal in $G$; so $\Theta(R)$ contains a maximal pair $(C, D)$ such that $C / D$ is Abelian. If $N \subseteq D$ then $(C / N, D / N)$ is a maximal pair in $\Theta(R / N)$ and $(C / N) /(D / N)$ is Abelian. If $N \not \subset D$, then 
if $N D \subset C, C / D$ will contain a proper normal subgroup of $G / D$; and if $C=N D$, then

$$
\langle R, C\rangle=G=\langle R, N D\rangle=R,
$$

a contradiction. We may therefore assume that $N \not \subset C$. Considering the pair $(C N, D N)$, one has that $C N / D N$ is Abelian. Let $K$ be the largest proper normal subgroup of $G$ contained in $C N$ such that $K \subseteq R$. If $C N / K$ does not contain any proper normal subgroup of $G / K$, then $(C N, K)$ is an element of $\Theta(R)$ and $(C, D) \leq(C N, K)$ implies that $C=C N$, a contradiction. If on the other hand $C N / K$ contains a proper normal subgroup of $G / K$, then suppose that $H / K$ is a minimal normal subgroup of $G / K$. Now, $H \subset C N, H \triangleleft G$, and $G=\langle R, H\rangle$. Therefore $(H, K)$ is a pair in $\Theta(R)$, and it is easy to see that $H / K$ is Abelian. If $(H, K)$ is a maximal pair, then $(H / N, K / N)$ is a maximal pair in $\Theta(R / N)$ and $(H / K) /(K / N)$ is Abelian. If on the other hand $(H, K)$ is not a maximal pair, then let $(H, K) \leq\left(H_{1}, K_{1}\right)$, where $\left(H_{1}, K_{1}\right)$ is a maximal pair and consequently $H \leq H_{1}$. One sees that $K_{1}$ is the largest proper normal subgroup of $G$ in $H_{1}$ that is contained in $R$; also $H$ is not contained in $K_{1}$. If $H K_{1} \neq H_{1}$, then $H K_{1} / H_{1}$ is a proper normal subgroup in $H_{1} / K_{1}$, a contradiction. Hence $H K_{1}=H_{1}$. It follows that $K \subset K_{1}$ and $H K_{1} / K_{1}$ is Abelian. Thus $\left(H_{1} / N, K_{1} / N\right)$ is a maximal pair in $\Theta(R / N)$ such that $\left(H_{1} / N\right) /\left(K_{1} / N\right)$ is Abelian. By induction, $G / N$ is solvable, and without loss in generality one may assume that $N$ is the unique minimal normal subgroup of $G$. If $N \subseteq L(G)$ (where $L(G)$ is defined in the statement of Theorem 3.2), then $G$ is solvable since $L(G)$ is supersolvable (a published proof appears in [3, Theorem 3]). Thus one may assume that $G=M N$, where $M$ is $c$-maximal and core-free in $G$. By hypothesis, there exists a maximal pair $(X,\langle 1\rangle)$ in $\Theta(M)$ such that $X /\langle 1\rangle$ is Abelian. If $X=N$ then $G$ is solvable. Let $\bar{X} \supset X$ and $X$ be a maximal subgroup of $\bar{X}$. If $\bar{X}$ does not contain any proper normal subgroup of $G$, then $(X,\langle 1\rangle) \leq(\bar{X},\langle 1\rangle)$ and $X=\bar{X}$, a contradiction. Thus $\bar{X}$ contains proper normal subgroup of $G$, and consequently $N \subset \bar{X}$. From a result of Huppert [6, Satz 2], it follows directly that if any group $Z$ has a maximal subgroup which is Abelian, then $Z$ is solvable. Since $\bar{X}$ has a maximal subgroup which is Abelian, it follows that $\bar{X}$ is solvable. So $N$ is solvable and consequently $G$ is solvable.

The converse holds trivially.

Theorem 3.4. If the index of each maximal pair in $\Theta(M)$ is a prime, for every $M \lessdot G$, then $G$ is supersolvable. (The index of a pair $(C, D)$ refers to $[C$ : $D])$.

Proof. If $G$ is simple, then the assertion follows trivially. Now, let $N$ be a maximal normal subgroup of $G$. We use induction on the order of $G$. If $M / N \lessdot G / N$ and $(X / N, Y / N)$ is a maximal pair in $\Theta(M / N)$, then by Lemma $2.1(X, Y)$ is a maximal pair in $\Theta(M)$ and the index of $(X / N, Y / N)$, being equal to $[X, Y]$, is a prime by using the hypothesis. By induction, $G / N$ is supersolvable and $N$ may be assumed to be the unique minimal normal 
subgroup of $G$. If $N \nsubseteq \Phi(G)$, then $G=M_{1} N$ for some core-free maximal subgroup $M_{1}$ of $G$. Then $(N,\langle 1\rangle)$ is a maximal pair in $\Theta\left(M_{1}\right)$ and by hypothesis $o(N)$ is a prime. Hence $G$ is supersolvable.

Corollary 3.5. A group $G$ is supersolvable $\Leftrightarrow$ the index of each maximal pair $(C, D)$ in $\Theta(M)$ is a prime whenever $C / D \triangleleft G / D$.

We omit the proof of the following result, which is analogous to Theorem 3.2(iii).

Proposition 3.6. A group $G$ is supersolvable $\Leftrightarrow$ for any two maximal subgroups $X, Y$ of $G$ whenever $\Theta(X)$ and $\Theta(Y)$ have a common maximal pair $(C, D)$, one has that $C / D$ is cyclic if $C / D \unlhd G / D$.

Let $Q(G)$ denote the characteristic subgroup of $G$ generated by all $x \in G$ with the property that

$$
\langle x\rangle \cdot\langle g\rangle=\langle g\rangle \cdot\langle x\rangle
$$

for every $g \in G$ (where as usual $\langle g\rangle$ denotes the subgroup generated by $g$ ). Let $Q^{*}(G)$ denote the terminal member in the ascending series defined by: $Q_{0}(G)=\langle 1\rangle, Q_{1}(G)=Q(G)$ and for $i>1$,

$$
Q_{i}\left(G / Q_{i-1}(G)\right)=Q_{i}(G) / Q_{i-1}(G) .
$$

These subgroups were introduced in Mukherjee [8]; also see [10] for an exposition.

Theorem 3.7. A group $G$ is supersolvable $\Leftrightarrow$ for every maximal pair $(C, D)$ in $\Theta(M)$, one has that $Q^{*}(G / D) \neq\langle\overline{1}\rangle$, for every $M \lessdot G$.

Proof. $\Leftarrow:$ It is obvious from the hypothesis that $G$ cannot be simple. Let $N$ be a minimal normal subgroup of $G$. We use induction on $o(G)$. As in the proof of Theorem 3.4, it follows that $G / N$ is supersolvable. Without loss in generality $N$ may now be assumed to be the unique minimal normal subgroup of $G, G=M N, M \lessdot G$; and $M$ is core-free. Then $(N,\langle 1\rangle)$ is a maximal pair in $\Theta(M)$, and therefore $Q^{*}(G) \neq\langle 1\rangle$. By the minimality of $N$, $N \subset Q^{*}(G)$. Therefore $N$ is a supersolvably embedded subgroup of $G$ and $o(N)$ is a prime $([10$, Theorem 7.10, p. 32]). Hence $G$ is supersolvable.

The converse holds trivially.

Corollary 3.8. A group $G$ is supersolvable if for every maximal pair $(C, D)$ in $\Theta(M)$ for each c-maximal subgroup $M$ of $G, Q^{*}(G) \neq\langle\overline{1}\rangle$.

\section{NilPOTENCY CONDITIONS}

Theorem 4.1. A group $G$ is nilpotent $\Leftrightarrow$ for each maximal pair $(C, D)$ in $\Theta(M)$ for every $M \lessdot G$, one has $Z(G / D) \neq\langle\overline{1}\rangle$.

Proof. $\Leftarrow$ : We use induction on $o(G)$ to show that every maximal subgroup of $G$ is normal. Clearly, $G$ cannot be simple. Let $M \lessdot G$ and $N$ be a minimal normal subgroup of $G$ contained in $M$. If $(C / N, D / N)$ is a maximal pair 
in $\Theta(M / N)$, then by Lemma 2.1, $(C, D)$ is a maximal pair in $\Theta(M)$ and $Z(G / D) \neq\langle\overline{1}\rangle$. This implies that $Z((G / N) /(D / N)) \neq\langle\overline{1}\rangle$. By induction, $M / N \triangleleft G / N$ and so $M \triangleleft G$. If $M$ is core-free, then a maximal pair in $\Theta(M)$ is of the form $(X,\langle 1\rangle)$ so that $Z(G) \neq\langle\overline{1}\rangle$. Thus $G=M Z(G)$ and therefore $M \triangleleft G$. The result now follows.

The converse holds trivially.

Corollary 4.2. A group $G$ is nilpotent $\Leftrightarrow \Theta(M)$, for every $M \lessdot G$, contains $a$ maximal pair $(C, D)$ such that $G / D$ is nilpotent.

Corollary 4.3. Let $M \lessdot G$ and $\Theta(M)$ contain a maximal pair such that $G / D$ is nilpotent. Then $M \triangleleft G$.

Theorem 4.4. A solvable group $G$, whose order is divisible by at least two primes, is nilpotent $\Leftrightarrow$ the index of each maximal pair in $\Theta(M)$, for every $M \lessdot G$, is the same.

Proof. $\Leftarrow$ : We use induction on the order of $G$. Let $N$ be a minimal normal subgroup of $G ; N$ is an elementary Abelian $p$-group for some prime $p$. By induction it follows that $G / N$ is nilpotent, and without loss in generality it may be assumed that $N$ is the unique minimal normal subgroup of $G$. If $N \nsubseteq \Phi(G)$, then for some $M \lessdot G, G=M N$ and $M$ is core-free. Now, $(N,\langle 1\rangle)$ is a maximal element in $\Theta(M)$, and so, by hypothesis, the index of any maximal pair in $\Theta(M)$ is a power of $p$. If $q$ is another divisor of the order of $G$, then all the elements of order $q$ in $G$ cannot lie in $M$, as they will generate a characteristic subgroup of $G$. Let $y \in G \backslash M$ such that $o(y)=q$. Then $\langle M, y\rangle=G$. If $\langle y\rangle \triangleleft G$, then $G=M\langle y\rangle$ and

$$
[G: M]=o(y)=q=o(N)=p^{m}
$$

for some $m \geq 1$, a contradiction to the fact that $q \neq p$. Thus we may assume that $\langle y\rangle$ is not normal in $G$. So $(\langle y\rangle,\langle 1\rangle) \in \Theta(M)$. Either $(\langle y\rangle,\langle 1\rangle)$ is a maximal pair in $\Theta(M)$, or if not, then $(\langle y\rangle,\langle 1\rangle) \leq(X,\langle 1\rangle)$, where $(X,\langle 1\rangle)$ is a maximal pair in $\Theta(M)$. But the index of $(X,\langle 1\rangle)$ is divisible by $q$, a contradiction. Thus $N \subseteq \Phi(G)$, and therefore $G$ is nilpotent.

The converse holds trivially.

We remark that Theorem 4.4 does not remain valid if in its statement one omits the condition that $G$ is solvable. For example, if $G$ is simple, then $(G,\langle 1\rangle)$ is the unique minimal pair in $\Theta(M)$ for every $M \lessdot G$, but $G$ is not nilpotent unless $o(G)$ is a prime.

Corollary 4.5. (i) A group $G$ is nilpotent $\Leftrightarrow$ the index of each maximal pair in $\Theta(M)$ for every $M \lessdot G$ is the same and is equal to a prime.

(ii) A solvable group $G$ is nilpotent $\Leftrightarrow \Theta(M)$, for all $M \lessdot G$, contains exactly one maximal pair.

We omit the proof of the following result. 
Proposition 4.6. A group $G$ is nilpotent $\Leftrightarrow$ for maximal pairs $(C, D)$ in $\Theta(M)$ and $\left(C_{1}, D_{1}\right)$ in $\Theta\left(M_{1}\right)$ with $C^{x}=C_{1}, M$ and $M_{1}$ are both normal in $G$.

For a group $G$ and a prime $p$, let $O^{p}(G)$ denote the subgroup generated by all the $p^{\prime}$-elements of $G$.

Theorem 4.7. Let $G$ be a group in which $\Theta(M)$, for each $M \lessdot G$, contains a maximal pair $(C, D)$ depending on $M$ such that

$$
O^{p}(G / D) \subseteq C_{G / D}(C / D)
$$

Then $G$ is p-nilpotent.

Proof. The assertion follows trivially if $G$ is simple. So, assume now that $G$ is not simple and let $N$ be a minimal normal subgroup of $G$. We use induction on $o(G)$. By inductive argument, it follows that $G / N$ is $p$-nilpotent; and one may also assume that $N$ is the unique minimal normal subgroup of $G$. If $N \subseteq \Phi(G)$, the assertion follows. So, let $G=M N$ for some core-free, maximal subgroup $M$ of $G$. By hypothesis, $\Theta(M)$ contains a maximal pair $(C, D)$ such that $C_{G}(G / D) \supseteq O^{p}(G / D)$. Then $D=\langle 1\rangle$ and $N \subseteq C_{G}\left(O^{p}(G)\right)$. Let $R / N$ be a normal $p$-complement in $G / N$. If $N$ is a $p^{\prime}$-group, then $R$ is a normal $p$-complement of $G$ and the assertion follows. Now, suppose that $p$ divides $o(N)$ Then, $N$ is an elementary Abelian $p$-group and is a normal Sylow $p$-subgroup of $R$. By the Schur-Zassenhaus theorem, $R=L_{0} N$, where $L_{0}$ is a $p$-complement of $N$. It is now easy to see that $L_{0}$ is a normal $p$-complement in $G$. Therefore $G$ is $p$-nilpotent.

For a group $G$ and a prime $p$, let $Z_{p^{\prime}}(G)$ denote the subgroup generated by all $x \in G$ which commute with every $p^{\prime}$-element of $G$. We omit the proof of the following result whose proof is analogous to that of Theorem 4.7.

Proposition 4.8. A group $G$ is p-nilpotent if for every $M \lessdot G, \Theta(M)$ contains a maximal pair $(C, D)$ such that $Z_{p^{\prime}}(G / D) \neq\langle\overline{1}\rangle$.

We now introduce two new characteristic subgroups of $G$ based on the concept of $\Theta$-pairs. Let

$$
\mathscr{F}=\{M<G: \exists \text { a maximal pair }(C, D) \in \Theta(M), Z(G / D)=\langle\overline{1}\rangle\} .
$$

Define $A(G)$ to be the intersection of all the elements of $\mathscr{F}$. Again, define $\mathscr{T}$ exactly in the same way as $\mathscr{F}$ is defined but only replacing the condition that " $Z(G / D)=\langle\overline{1}\rangle$ " by the condition that " $Q^{*}(G)=\langle 1\rangle$ ". Define $B(G)$ to be the intersection of all the elements of $\mathscr{T}$. (If either of the families $\mathscr{F}, \mathscr{T}$ is empty, the corresponding subgroup is chosen to be $G$ itself).

The proof of the following result is left as an exercise.

Proposition 4.9. (i) $A(G)$ is a characteristic subgroup of $G$ which is nilpotent, and $A / \Phi(G)=Z_{\infty}(G / \Phi(G))$.

(ii) $B(G)$ is a characteristic subgroup of $G$, and $B(G)$ is supersolvable. 


\section{REFERENCES}

1. R. Baer, Classes of finite groups and their properties, Illinois J. Math. 1 (1957), 115-187.

2. H. C. Bhatia, A generalized Frattini subgroup of a finite group, Ph. D. thesis, Michigan State Univ., East Lansing, MI, 1972.

3. P. Bhattacharya and N. P. Mukherjee, On the intersection of a class of maximal subgroups of a finite group II, J. Pure Appl. Algebra 42 (1986), 117-124.

4. W. E. Deskins, On maximal subgroups, Proc. Sympos. Pure Math. 1 (1959), 100-104.

5. __ A note on the index complex of a maximal subgroup, Arch. Math. (to appear).

6. B. Huppert, Normalteiler und maximale Untergruppen endlicher Gruppen, Math. Z. 60 (1954), 409-434.

7. __ Endliche Gruppen I, Springer-Verlag, Berlin, 1967.

8. N. P. Mukherjee, The hyperquasicenter of a finite group I, II, Proc. Amer. Math. Soc. 26 (1970), 239-243; 32 (1972), 24-28.

9. N. P. Mukherjee and P. Bhattacharya, The normal index of a maximal subgroup of a finite group, Proc. Amer. Math. Soc. 106 (1989), 25-32.

10. M. Weinstein (ed.), Between nilpotent and solvable, Polygonal Publ., Washington, NJ, 1982.

School of Computer and System Sciences, Jawaharlal Nehru University, New Delhi 110067 , INDIA

Department of Computer SCIENCe ANd Engineering, University of Nebraska-Lincoln, LiNCOLN, NEBRASKa 68588-0115 